\title{
Suicidal Attempt With Intentional Poisoning Seems a Comorbid Illness With an Increased Burden
}

\author{
Reza Assadi ${ }^{1}$ and Reza Afshari ${ }^{2,3,{ }^{*}}$ \\ ${ }^{1}$ Medical Toxicology Research Center, Mashhad University of Medical Sciences, Mashhad, IR Iran \\ ${ }^{2}$ Addiction Research Center, Imam Reza Hospital, Mashhad University of Medical Sciences, Mashhad, IR Iran \\ ${ }^{3}$ BC Disease Control Center, Vancouver, Canada \\ *Corresponding author: Reza Afshari, Addiction Research Center, Imam Reza Hospital, Ibn-Sina St. Mashhad, IR Iran. Tel: +98-5138525315, Fax:+98-5138525315, E-mail: afsharir@mums.ac.ir
}

Received 2014 November 5; Revised 2015 February 21; Accepted 2015 April 8.

\begin{abstract}
Background: In measuring health utilities, the primary reason for selecting patients as a source for valuations is that they directly experience the impact of the disease.

Objectives: Accordingly, the aim of this study was to examine the variation in generic utility measures with respect to acute poisonings by including a comparison between those subjects who had high intention and low intention to commit suicide.

Patients and Methods: We evaluated the responses of patients who had attempted suicide and were admitted to a toxicology ward. We used multiple methods, including TTO, VAS, and EQ-5D.

Results: We reviewed the collected questionnaires of one hundred patients admitted to the medical toxicology ward of Emam Reza teaching hospital in Mashhad, Iran. Our results show that the mental state after an incomplete suicide attempt can present either a real desire for suicide or a desire for attention from relatives and rejection of life problems.

Conclusions: This study demonstrates that the mental states associated with specific diseases should not be ignored in evaluating health states. Although there are benefits to relying on expert panels and the general population in evaluating various health states, attention to the particular health states of the patients (taking into account their associated mental well-being) should also be utilized.
\end{abstract}

Keywords: Acute Disease, Attempted, Illness Burdens, Global Health, Life Tables, Life Expectancy, Models, Statistical, Mortality, Poisoning, Suicide

\section{Background}

In health economics, measuring health utilities involves two main steps: defining a set of health states of interest and assigning values to those health states. There are both direct and indirect methods of utility valuation. Some of the methods that have been used to collect data on utilities are the standard gamble (SG) approach, the time trade-off (TTO) approach, and the visual analogue (VAS) approach. The valuation of preferences using the SG, TTO, or VAS can be performed by the public, patients, or experts (1).

The rationale for measuring the preferences of the general public for hypothetical health states is that it is society's resources that are being allocated in a publically funded health system (2). The advantage of the patientcentered approach over a hypothetical approach is that, although it is more challenging to recruit patients with the specific health states of interest, utility measures obtained through hypothetical scenarios may not be valid predictors of preferences associated with actual experienced health states (3). There are also differences between the reported values of each method; in general, patient values for hypothetical health states are likely to be worse than their current health state but tend to be higher than those obtained from the general public for that particular condition (3).

van Spijker et al. measured the health states caused by suicidal thinking and non-fatal suicide attempts. They concluded that suicidal thoughts are as disabling as alcohol dependence and severe asthma. The mental distress involved in non-fatal suicide attempts is thought to be comparable in level of disability to heroin dependence and initial stage Parkinson's disease. These results demonstrate the severity of suicidality on health (4).

One common form of suicide is poisoning. Poisoning is a disease with acute or chronic onset. Acute onset poisonings happen due to intentional, unintentional, and criminal misuse of toxic substances or medicines. In the current study we examined the valuations of patients who attempted suicide or expressed a desire to end their life by poisoning. Our initial investigation confirmed the presence of two classes of patients among those admitted to clinical toxicology services due to self-harm actions. The first group includes those whose suicide attempt does not reflect a deep commitment to dying (the low-intention group). The second group (the high-inten-

Copyright (C) 2016, Zahedan University of Medical Sciences. This is an open-access article distributed under the terms of the Creative Commons Attribution-NonCommercial 4.0 International License (http://creativecommons.org/licenses/by-nc/4.0/) which permits copy and redistribute the material just in noncommercial usages, provided the original work is properly cited. 
tion group) includes subjects who had a deep desire to die and did not just use higher doses, but also more lethal medications and poisons, than the low-intention group. Even after recovery, this group valued their condition less than the other group.

In measuring health utility, it is common to utilize the Burden of Disease (GBD), a study presented in 1990, which quantified the health effects of more than one hundred diseases and injuries for eight regions of the world (5-7). This study also introduced a new metric, the disabilityadjusted life year (DALY), as a single measure for quantifying the burden of diseases, injuries, and risk factors. The DALY is based on years of life lost from premature death and years of life lived in less-than-full health. In this new measure, disability weights (DWs) were designated for various health problems based on various health valuation methods $(1,5)$. These indices have been subject to severe debate, as well as several studies (6).

Although the GBD study has been updated several times by the World Health Organization (WHO), a single range has always been used for poisonings (8). Nevertheless, it would seem that the subjects of poisonings should have various health states based on substance, condition, and demographics. In the new Global Burden of Disease study undertaken by WHO in 2004 , poisoning diseases were scored between 0.608 to 0.611 for those under and above 14 years old, respectively (5). The weight was even decreased to .131 in the recent publication (9).

The methods of health valuation used by the world health organization consisted of various direct and indirect methods. Among the direct methods were time trade-off, person trade-off, standard gamble, and EQ-5D. In the case of acute disease, non-experts were used for determining the time trade-off and VAS (using EQ-5D). This was the method used to evaluate the health state of poisoned patients (1). However, in the current study, in the course of the data collection we encountered a lack of commitment or desire to live in the patients. So, it was hypothesized that in some cases the mental states of the respondents play a role in the value assigned for health and disease in this particular health problem $(1,10)$.

\section{Objectives}

The aim of this study was to compare the variation in the generic utility measures of intentional acute poisonings to patients' willingness to live and their mental state after recovering from their suicide attempt.

\section{Patients and Methods}

In the current study we evaluated the responses of patients who had attempted suicide and were admitted to a toxicology ward. We used multiple methods, including TTO, VAS, and EQ-5D. We aimed to determine how a patient with a generally poor perception about living and self would evaluate their life under such conditions and how this value score might differ from those in the same condition of physical health but without suicidality. We reviewed the data from questionnaires administered to one hundred patients admitted to the medical toxicology ward of Emam Reza teaching hospital in Mashhad, Iran. In these questionnaires, the patients provided information about the substance used, cause of consumption (e.g., intentional, unintentional, or criminal), the main challenge of attempting suicide, and the time of the suicide attempt. Also included were VAS, TTO, and EQ-5D scores. The demographic information of the patients was also collected. The patients were divided into two groups based on their high or low intention to die after the first incomplete suicide attempt. This item was obtained based on the patient's own expression and the psychiatric consult concerning the risk of a repeated attempt.

Those who were unconscious, had severe psychological disorders (e.g., depression or psychosis), intensive care unit admission, criminal suspicion of poisoning, a lack of collaboration with inquirers, or literacy levels that precluded understanding of the valuation technique were excluded from the study.

\subsection{EQ-5D Profiles}

One of the main techniques used for health state evaluation was developed by the EuroQoL Group. An EQ-5D health state (or profile) is a set of observations about a person's health, defined by a descriptive system. An EQ-5D health state may be converted to a single summary index by applying a formula that essentially attaches weights to each of the levels in each dimension. This formula is based on the valuation of EQ-5D health states from general population samples (11). The standardized extended version of the EQ5D was designed for the collection of health state values using a five-question rating scale. Mobility, activity, self-care, worry, and pain or discomfort are all judged on a three-step scale, varying from no problem to lots of problems. In this method, each answer is a number from 1 to 3 , with the best health measured as 11111 and the worst health as 33333. This valuation method is based on patient answers and results in 243 health states (12). This questionnaire has been translated by the EuroQol Group into various languages, and for this study, after filling out a form about the current study method on the group's website, the translated and validated version was sent to the participants.

\subsection{Statistical Analysis}

In the current study the descriptive information of the subjects was identified and presented in terms of mean \pm SD. The correlation between the EQ-5D sub-items was evaluated using an $\chi^{2}$ test. In all statistical analyses, a Pvalue less than .05 was considered significant.

\section{Results}

Consenting respondents completed an EQ-5D questionnaire describing their own health using EQ-5D, EQ-VAS, 
and TTO. In the current study, we collected data of one hundred patients admitted to the medical toxicology service of Imam Reza teaching hospital in Mashhad, Iran. The mean age of the sample was $30.12 \pm 15$, with a range of 16 to 50 years old. The male to female ratio was 1.2:1.0. Most of the patients were literate but without higher education qualifications. Detailed demographics are shown in Table 1. The most common substances used for poisoning were benzodiazepines, opiates, salicylates, organophosphates, and psychotherapeutic agents.

Among the patients, 82 cases were eligible to enter the study based on consent, the type of poisoning, and the appropriateness of the responses. Of the sample, 54\% were female and $46 \%$ were male. Among all participants, we found that $26 \%$ had a history of former suicide attempts. The causes of suicide attempts and the abused substance used are shown in Table 2.

The mean scores of the EQ-5D-3L for patients in both groups are shown in Table 3, which compares the differences in the patients based on their intention for committing suicide or not. Also, as shown in Table 1, there was no significant difference in the EQ-5D scores of the patients in both groups. This similarity also held true for the VAS section of the questionnaire. However, in the case of TTO responses, the patients of the first group (those with a desire to live or low-intention suicide group) scored a mean trade-off time of five years. In contrast, in the other group, $66 \%$ did not score their health and did not trade off anything (their life being viewed as not worth living). Those who did suggest a trade-off had a mean score of 20 years. This is significantly higher than the first group (Table 3 ).

\begin{tabular}{|c|c|}
\hline Variable/Subtype & Values \\
\hline \multicolumn{2}{|l|}{ Age, $y$} \\
\hline$>18$ & $10(12)$ \\
\hline $19-35$ & $42(51)$ \\
\hline$<36$ & $30(36)$ \\
\hline \multicolumn{2}{|l|}{ Marital status } \\
\hline Single & $15(18)$ \\
\hline Married & $65(79)$ \\
\hline Divorced/Widowed & $12(14)$ \\
\hline \multicolumn{2}{|l|}{ Gender } \\
\hline Male & $34(41)$ \\
\hline Female & $48(58)$ \\
\hline \multicolumn{2}{|l|}{ Cause of poisoning } \\
\hline Familial conflict & $50(60)$ \\
\hline Psychological & $22(26)$ \\
\hline Business conflict & $6(7.3)$ \\
\hline Criminal & $4(4.8)$ \\
\hline \multicolumn{2}{|c|}{ Addiction to abused substance } \\
\hline Yes & $22(26)$ \\
\hline No & $50(60)$ \\
\hline Unresponsive & $10(12)$ \\
\hline \multicolumn{2}{|c|}{ Time of suicidal attempt } \\
\hline First & $60(75)$ \\
\hline Second or more & $22(26)$ \\
\hline \multicolumn{2}{|c|}{ Psychological problem } \\
\hline Yes & $26(31)$ \\
\hline No & $56(68)$ \\
\hline \multicolumn{2}{|l|}{ Cause of poisoning } \\
\hline Intentional & $26(31)$ \\
\hline Unintentional & $54(65)$ \\
\hline Criminal & $2(2.4)$ \\
\hline
\end{tabular}


Assadi R et al.

Table 2. An Overview of the Mean EQ Sub-Item Ratings and the Proportions of Reported Problems on Each of the 5 EQ-5D Dimensions Between the Two Groups of Patients who Underwent Intentional Substance Abuse

\begin{tabular}{lccc}
\hline EQ-5D-3L Sub-Items & Patients Desired to Die & Patients Desired to Obtain Gains & P Value \\
\hline Mobility & 32 & 45 & $>0.05$ \\
Doing usual activities & 30 & 60 & $>0.05$ \\
Caring own & 33 & 51 & $>0.05$ \\
Pain/discomfort & 41 & 57 & $>0.05$ \\
Being anxious/worry & 51 & 54 & $>0.05$ \\
\hline
\end{tabular}

\begin{tabular}{lccc}
\hline \multicolumn{2}{l}{ Table 3. The Mean Scores of TTO and VAS Among Three Groups of the Patients } & TTO Score Mean & VAS Score Mean \\
\hline \multicolumn{1}{l}{ Cause of Poisoning } & No. (\%) & & $40^{\mathrm{a}}$ \\
\hline Intentional & $12(15)$ & 5.1 & 61 \\
$\quad$ Intended to die & $46(56)$ & 5.8 & 81 \\
\hline Intended temporary gains & $24(29)$ & & 45 \\
\hline Unintentional
\end{tabular}

${ }^{\mathrm{a}}$ While $66 \%$ of the participants in this group did not trade off any years as they counted their life meaningless.

\section{Discussion}

This study is part of the first known epidemiological project attempting to evaluate the health states of those intending to die by intentional abuse of toxic substances. It also compares the scores of the subjects with those poisoned due to unintentional abuse or intentional abuse with/without a real desire to die (high or low intention). We endeavored to see how the real desire to end life affects the health perception of these patients. Although there are arguments for rejecting the patients' own perceptions as a source of valuation (1), our findings demonstrate that they should not be ignored because of the specific nature of mental states and manifestations of the disease. A person's mental state after an incomplete suicide attempt can reveal either a real desire to die, or that they have been seeking the attention of relatives or rejecting their own problems. Some patients really aim to end their lives and may undertake more than one suicide attempt without changing their minds. For the group of patients with low intention, our findings show a health value almost as high as the general score for poisoning reported by the WHO's Global Burden of Disease. However, the high-intention group placed no value on their health, so it might be better to assign a higher score to this group.

In our study there was no statistical difference between the EQ-5D scores of the patients and the proportion of the patients who reported a problem in any of the five dimensions in both groups. This was observed despite differences in TTO or the amount of time they traded off. This means that the first group traded off less time for their health valuation (Table 3), while the second group scored their general health situation as even worse than the group who did not really desire to die after an incomplete attempt. This group was the majority of those expressing their willingness to repeat their attempt to end their life, after the first incomplete attempt.

In 1998 , suicide was responsible for $1.8 \%$ of the total burden of disease worldwide, varying from $2.3 \%$ in highincome countries to $1.7 \%$ in low-income countries (4). Unfortunately, little data is available on the recent DALYs due to suicide in Iran, because the national disease burden study was published in 2003 (13), but it seems that rates are increasing.

Nevertheless, suicide is considered a health problem with consequences beyond the disability of the individual. On average, a single suicide intimately affects at least six other people. If a suicide occurs in a school or workplace, there is an impact on hundreds of people. In the case of health valuation and measuring techniques, it would seem that, despite the substance used and even perhaps the method used for suicide, a person who really desires to end their life may have a burden much larger than what is measured (4).

Calculating the burden of disease for suicide in DALYs puts suicide twenty-first in the list of the most important diseases in the Netherlands. Non-fatal suicide ranks worse, due to mental and physical suffering. Other literature reviews have considered the resulting DWs for suicidal thoughts (0.36) as parallel to the DWs for related psychiatric disorders. Suicidal thoughts are considered as disabling as alcohol dependence (0.32) and cocaine dependence (0.33) (14). The data for these studies are gathered from expert panel reviews. The findings of our study further show that suicidal patients are of two types: low and high intention. The value these groups assign to their health is generally different. Some patients admitted to the poisoning department really desire to die (high intention), but some others lack the intention to repeat a suicide attempt after the first trial (low inten- 
tion). Their behaviour is also a cause of anxiety and tension for relatives and others in close contact with them. This issue is what is ignored in almost all studies ranking of the health states of these patients. Similarly, suicidal thoughts and attempts are likely to be underestimated when the person performing the rating does not have access to the sufferer's mind (14).

So, while to our knowledge it is not clear that those who commit suicide will receive one of the DW codes of 0.608 for poisoning or 0.48 for suicide, our suggestion is to consider those with high intention of suicide as having a comorbid condition. This means their disability score should be s combination of poisoning and suicide attempt. The below formula is a method for comorbid disability estimation with an example (DW for poisoning is 0.608 and DW for suicide is 0.48 ):

$\mathrm{DW}=\left(\mathrm{DW}_{1}\right) \times 1+\left(\mathrm{DW}_{2} \times 1-\mathrm{DW}_{1}\right)$

This calculation increases the DW of poisoning and suicidal attempt to a comorbid condition that seems more parallel to our findings in this study.

While there are benefits to relying on expert panels and the general population for valuation of various health states, attention to the particular health states and the mental wellbeing of the person suffering from a health condition also might be useful for better understanding of the health state of the patients and might demonstrate an increased burden that was not shown by other methods (15-17).

\section{Acknowledgments}

We are thankful from MUMS and all of our patients.

\section{Footnotes}

Authors' Contribution:Reza Assadi has collected data and prepared the draft of the paper. Reza Afshari is the supervisor of the aforementioned project and has proofread the paper.

Funding/Support:Mashhad University of Medical Sciences.

\section{References}

1. Mphil KT. What are health utilities? 2009. Available from: http:// www.medicine.ox.ac.uk/bandolier/painres/download/whatis/ Health-util.pdf.
2. Rehm J, Frick U. Establishing disability weights from pairwise comparisons for a US burden of disease study. Int J Methods Psychiatr Res. 2013;22(2):144-54. doi: 10.1002/mpr.1383. [PubMed: 23716479]

3. Kuspinar A, Mayo NE. Do generic utility measures capture what is important to the quality of life of people with multiple sclerosis? Health Qual Life Outcomes. 2013;11:71. doi: 10.1186/1477-7525-1171. [PubMed: 23618072]

4. van Spijker BA, van Straten A, Kerkhof AJ, Hoeymans N, Smit F. Disability weights for suicidal thoughts and non-fatal suicide attempts. J Affect Disord. 2011;134(1-3):341-7. doi: 10.1016/j. jad.2011.05.020. [PubMed: 21652085]

5. World health organization. The global burden of disease: 2004 update. 2004. Available from: http://www.who.int/healthinfo/ global_burden_disease/2004_report_update/en/.

6. Murray CJ, Mathers CD, Salomon JA, Lopez AD. Health gaps: an overview and critical appraisal. WHO publishing. 2002.

7. Rabin R, de Charro F. EQ-5D: a measure of health status from the EuroQol Group. Ann Med. 2001;33(5):337-43. [PubMed: 11491192]

8. Murray CJ, Vos T, Lozano R, Naghavi M, Flaxman AD, Michaud C, et al. Disability-adjusted life years (DALYs) for 291 diseases and injuries in 21 regions, 1990-2010: a systematic analysis for the Global Burden of Disease Study 2010. Lancet. 2012;380(9859):2197-223. doi:10.1016/S0140-6736(12)61689-4. [PubMed: 23245608]

9. Salomon JA, Vos T, Hogan DR, Gagnon M, Naghavi M, Mokdad A, et al. Common values in assessing health outcomes from disease and injury: disability weights measurement study for the Global Burden of Disease Study 2010. Lancet. 2012;380(9859):2129-43. doi:10.1016/S0140-6736(12)61680-8. [PubMed: 23245605]

10. Schwarzinger M, Stouthard ME, Burstrom K, Nord E. Cross-national agreement on disability weights: the European Disability Weights Project. Popul Health Metr. 2003;1(1):9. doi: 10.1186/14787954-1-9. [PubMed:14633276]

11. Scalone L, Cortesi PA, Ciampichini R, Belisari A, D'Angiolella LS, Cesana G, et al. Italian population-based values of EQ-5D health states. Value Health. 2013;16(5):814-22. doi: 10.1016/j. jval.2013.04.008. [PubMed: 23947975]

12. EuroQol. Valuation of EQ-5D. 2013. Available from: http://www.euroqol.org/about-eq-5d/valuation-of-eq-5d.html.

13. Naghavi M, Abolhassani F, Pourmalek F, Lakeh M, Jafari N, Vaseghi S, et al. The burden of disease and injury in Iran 2003. Popul Health Metr. 2009;7:9. doi:10.1186/1478-7954-7-9. [PubMed: 19527516]

14. Whiteford HA, Degenhardt L, Rehm J, Baxter AJ, Ferrari AJ, Erskine HE, et al. Global burden of disease attributable to mental and substance use disorders: findings from the Global Burden of Disease Study 2010. Lancet. 2013;382(9904):1575-86. doi: 10.1016| S0140-6736(13)61611-6. [PubMed: 23993280]

15. Sullivan PW, Slejko JF, Sculpher MJ, Ghushchyan V. Catalogue of EQ-5D Scores for the United Kingdom. Med Decision Making. 2011;31(6):800-4. doi:10.1177/0272989x11401031.

16. Afshari R, Majdzadeh R, Balali-Mood M. Pattern of acute poisonings in Mashhad, Iran 1993-2000. J Toxicol Clin Toxicol. 2004;42(7):965-75. [PubMed:15641642]

17. Chapman BP, Franks P, Duberstein PR, Jerant A. Differences between individual and societal health state valuations: any link with personality? Med Care. 2009;47(8):902. [PubMed: 19543121] 